$\xi=-1$ 圆

\title{
A survery on platform of secured routing algorithm
}

\author{
Miss. Divya ${ }^{1}$, Dr. R. Gobinath ${ }^{2}$ \\ ${ }^{1}$ Research Scholar, Department of Computer Science, Vels Institute of Science, Technology and Advanced Studies (VISTAS), Pallava- \\ ram, Chennai, Assistant Professor, Department of Computer Science, Prince Shri Venkateshwara Arts and Science College-Chennai. \\ ${ }^{2}$ Assistant Professor, Department of Computer Science, VISTAS, Pallavaram, Chennai. \\ *Corresponding author E-mail: iamgobinathmca@ gmail.com
}

\begin{abstract}
This paper describes the characteristics and techniques of Ad Hoc network. Ad Hoc networks are temporary network, set anywhere without any need of external infrastructure like wires. The essential premise of this paper is to exchange confidential message over wireless medium without route break and unauthorized eavesdroppers. In recent years, the research of routing protocol for AdHoc network has caused public concern widely. The multi hopping routing protocol has failed to meet the needs of network topology and route restructure. It must also consider the bandwidth and high error rate. The new routing protocol suitable for solving these problems in Ad Hoc network is AODV.
\end{abstract}

Keywords: Ad Hoc Network; Network Security; Routing; AODV Routing Protocol

\section{Introduction}

A computer network is a gathering of computer systems and other computing hardware resources that are allied together through communication channels to promote transmission and resourcesharing amid a broad area of users. Networks are frequently assorted based on their aspects. Computer networks support a vast number of relevance and services such as approach to the World Wide Web, digital video, digital audio, shared use of application and storage servers, printers, and fax machines, and use of email and instant messaging applications as well as many others. Computer networks vary in the communication medium used to transfer their signals, communications protocols to formulate network jam, the network's size, topology and organizational intent. The prime familiar computer network is the Internet.Computer communication junction carries data as a bit flood. However, maximum messages in computer networks are toted in packets. A network packet is a patterned unit of data (a list of bits or bytes, usually a few tens of bytes to a few kilobytes long) transmitted by a packet-switched network. In packet networks, the data is patterned into packets that are directed through the network to their target. Once the packets reach they are gathered into their initial message. In communication networks, a node is either a redistribution point or a communication endpoint. A physical network node is an operative computerized device that is connected to a network, and is efficient of creating, receiving, or transmitting messages over a transmission channel. In telecommunications, data transfer between the node-to-node is the flow of data from one node of a network to the next. In the OSI model it is managed by the least two layers, the data link layer and the physical layer. Routing determines good passage through a network to carry the message from source to target. Routing is the manner of choosing network paths to carry network jam.

\section{Review of literature}

An implementation study of AODV routing protocol [6] express the evolution in AODV protocol. The AODV is implemented in Linux Kernel because of its implicit portability and wide source nature of Linux. AODV protocol manages an orbital timer for updating multicast route table access. Security Enhancements in AODV protocol for wireless Ad hoc networks [7] withstands certain misbehavior provoked by malign node for AODV routing protocol. This paper proposes the security schemes to prevent the internal attacks. An Intrusion Detection and Intrusion Prevention method is also discussed to prevent several attacks in network. AODV with Path Accumulation [8] examines the expected alterations by organizing the path accumulation feature in AODV. The texture of protocol helps to minimize the routing capacity and sustain excellent performance. This paper introduces the advanced protocol AODV-PA to enhance the performance. The AODV-PA (path accumulation) aids to minimize the number of route discoveries than AODV. Multi - linked AODV Routing protocol for wireless Mesh network [9] symbolizes the modified AODV protocol called AODV-ML (multi link). This protocol successfully observes the bidirectional channel between neighboring nodes in multi hop wireless network. This work achieves high stable of efficiency by acquiring reduced overhead. High performance AODV routing protocol for Hybrid wireless Mesh Network [10] instants an advanced protocol called AODV-HP (hybrid protocol) which is derived from AODV protocol. This protocol ease the issue associated with the physical, MAC and network layer in hybrid WMNs. Combat with Black Hole attack in AODV routing protocol in MANET [11] demonstrates MAODV schema which is used to combat black hole attack in AODV routing protocol. This paper also reviews the security problem of MANET. Global Mobile Simulator is used to gain the prescribed security with limited delay and overhead. 
Bypass AODV: Improving Performance of Ad hoc On-demand Distance Vector (AODV) routing protocol in wireless Ad hoc Networks [12] enumerates that Bypass-AODV is derived to enhance the utilization of AODV routing protocol. Bypass-AODV adopts an explicit strategy of cross-layer MAC-interaction to establish mobility-related route breaks. This paper also illustrates the encounter on node density and portability of Bypass-AODV.A dynamic Anomaly Detection scheme for AODV - Based mobile Ad hoc networks [13] reports a new dynamic anomaly detection technique for MANET. For elevating the security robust learning techniques are used. Misuse detection and Anomaly detection techniques are recycled for identifying malicious attacks. In Anomaly detection method the packet carried at each node is inspected.

Performance evaluation of energy consumption for AODV and DSR routing protocols in MANET [14] inspect the valuation of energy consumption of AODV routing protocol. The operation of this research comprises simulation scenario, portability arrangement and energy arrangement. AODV protocol endures route discovery and route maintenance to manage routing information. An Energy efficient routing protocol decreases the power consumption of the nodes. Research of AODV routing protocol for Ad hoc networks [15] characterize the basic properties of Ad hoc

network. An AODV protocol represents an optimized protocol called B-AODV established on shortage of route finding and routing reformation on AODV. This paper also abstracts the performance of routing protocol build upon routing overhead, node delay, packet success rate and so on. The prime protocol upgrades the route reformation capacity and minimizes the routing overhead. The above table describes the list of measurements taken in secured Ad HOC networks. These works specifies the list of methods and tech-niques used to transfer the data securely in the wireless network environment. The table also mentions how different Authors used various security algorithms to solve the network security issues. The above table discusses various applications of AODV routing protocol and also describes how various authors used this protocol to solve the problem of route break in network routing while transmitting the valuable data over secured wireless network. The TABLE II also explains about various measurements observed by different authors in AODV routing protocol with network security.

\section{Existing work}

Table 1: Measurements Taken in Network Security

\begin{tabular}{|c|c|c|c|c|c|}
\hline SNO & YEAR & AUTHOR & TOPIC & MEASUREMENS & DESCRIPTION \\
\hline 1 & DEC 1999 & $\begin{array}{l}\text { Lidong Zhou, } \\
\text { Et al. }\end{array}$ & Securing Ad hoc Networks & $\begin{array}{l}\text { Threshold } \\
\text { Secrecy }\end{array}$ & $\begin{array}{l}\text { This paper studies about the } \\
\text { network security issues using } \\
\text { multiple routing techniques in } \\
\text { cryptography. }\end{array}$ \\
\hline 2 & MAR 2000 & $\begin{array}{l}\text { Piyush Gupta, } \\
\text { Et al. }\end{array}$ & The capacity of wireless networks & $\begin{array}{l}\text { Throughput } \\
\& \\
\text { Capacity }\end{array}$ & $\begin{array}{l}\text { This paper investigates the } \\
\text { network security level in } \\
\text { ALOHA network using } \\
\text { TDMA, FDMA \& CDMA. }\end{array}$ \\
\hline 3 & 2005 & $\begin{array}{l}\text { Michael } \\
\text { J. Neely, } \\
\text { Et al. }\end{array}$ & $\begin{array}{l}\text { Capacity and Delay Trade-offs } \\
\text { for Ad Hoc Networks }\end{array}$ & $\begin{array}{l}\text { Capacity } \\
\& \\
\text { Delay }\end{array}$ & $\begin{array}{l}\text { This paper describes the prob- } \\
\text { lem } \\
\text { of security in cell partitioning } \\
\text { network using two hop relay }\end{array}$ \\
\hline 4 & 2005 & Patrick Herhold, et al. & $\begin{array}{l}\text { Cooperative Multi hop } \\
\text { Transmission in wireless network }\end{array}$ & Capacity & $\begin{array}{l}\text { This paper studies the issues of } \\
\text { network security in two hop } \\
\text { algorithm and provides secured } \\
\text { data transmission using multi } \\
\text { hopping. }\end{array}$ \\
\hline 5 & SEP 2007 & ShabnamShafiee, et al. & $\begin{array}{l}\text { Secrecy Capacity of Gaussian } \\
\text { MIMO Wire-tap channel }\end{array}$ & $\begin{array}{l}\text { Secrecy capacity, Secrecy } \\
\text { rate } \\
\& \\
\text { Power Allocation }\end{array}$ & $\begin{array}{l}\text { This paper investigates secured } \\
\text { data communication in MIMO } \\
\text { wiretap channel using multiple } \\
\text { antenna system in cryptograph- } \\
\text { ic methods. }\end{array}$ \\
\hline 6 & 2008 & F. Oggier, et al. & The MIMO Wiretap Channel & $\begin{array}{l}\text { Equivocation rate, secrecy } \\
\text { rate } \\
\& \\
\text { Secrecy capacity }\end{array}$ & $\begin{array}{l}\text { This paper describes the issues } \\
\text { in single antenna system and } \\
\text { provides solution using multi- } \\
\text { ple antenna channels in Infor- } \\
\text { mation Theoretic System. }\end{array}$ \\
\hline 7 & OCT 2008 & $\begin{array}{l}\text { OnurOzanKoylough, et } \\
\text { al. }\end{array}$ & $\begin{array}{l}\text { Interference Alignment for Secre- } \\
\text { cy }\end{array}$ & $\begin{array}{l}\text { Equivocation rate, Secrecy } \\
\text { rate } \\
\& \\
\text { Secrecy capacity }\end{array}$ & $\begin{array}{l}\text { This paper explains the secured } \\
\text { data transmission using wiretap } \\
\text { channels in Information Theo- } \\
\text { retic System. }\end{array}$ \\
\hline 8 & MAR 2010 & $\begin{array}{l}\text { S. Anand, } \\
\text { R.Chandra-mouli } \\
\text { (IEEE Members) }\end{array}$ & $\begin{array}{l}\text { On the Location of an Eaves- } \\
\text { droppers in multi terminal net- } \\
\text { works }\end{array}$ & $\begin{array}{l}\text { Secrecy capacity, Power } \\
\text { allocation }\end{array}$ & $\begin{array}{l}\text { This paper studies about net- } \\
\text { work security issues in crypto- } \\
\text { graphic methods using artifi- } \\
\text { cial noise generation in Infor- } \\
\text { mation Theoretic System }\end{array}$ \\
\hline 9 & JUN 2010 & $\begin{array}{l}\text { Xiangyunzhou } \\
\text { et al. }\end{array}$ & $\begin{array}{l}\text { Secure Transmission with Artifi- } \\
\text { cial Noise over Fading Channels }\end{array}$ & $\begin{array}{l}\text { Capacity, } \\
\text { Power Allocation }\end{array}$ & $\begin{array}{l}\text { This paper investigates the } \\
\text { network security problem } \\
\text { during data transmission using } \\
\text { cryptographic methods. }\end{array}$ \\
\hline 10 & JUN 2010 & $\begin{array}{l}\text { AshishKhisti, } \\
\text { Et al. }\end{array}$ & $\begin{array}{l}\text { Secure Transmission with the } \\
\text { MIMOME Wiretap Channel }\end{array}$ & $\begin{array}{l}\text { Secrecy capacity, Through- } \\
\text { put }\end{array}$ & $\begin{array}{l}\text { This paper describes the issues } \\
\text { in secured data transmission } \\
\text { using multiple antenna system }\end{array}$ \\
\hline 11 & FEB 2011 & $\begin{array}{l}\text { Pedro C. Pinto, } \\
\text { et al. }\end{array}$ & $\begin{array}{l}\text { Wireless Secrecy in Large Scale } \\
\text { Networks }\end{array}$ & $\begin{array}{l}\text { Precision, } \\
\text { Degree } \\
\& \\
\text { Secrecy rate }\end{array}$ & $\begin{array}{l}\text { This paper explains the issues } \\
\text { of wireless network security } \\
\text { using Information Theoretic } \\
\text { System. }\end{array}$ \\
\hline 12 & APR 2011 & $\begin{array}{l}\text { Jing Huang, } \\
\text { Et al. }\end{array}$ & $\begin{array}{l}\text { Robust Secure Transmission in } \\
\text { MISO } \\
\text { Channels based on Worst-case }\end{array}$ & $\begin{array}{l}\text { Secrecy rate, } \\
\text { Power allocation }\end{array}$ & $\begin{array}{l}\text { This paper investigates the } \\
\text { problem of secured data trans- } \\
\text { mission in cryptography using }\end{array}$ \\
\hline
\end{tabular}




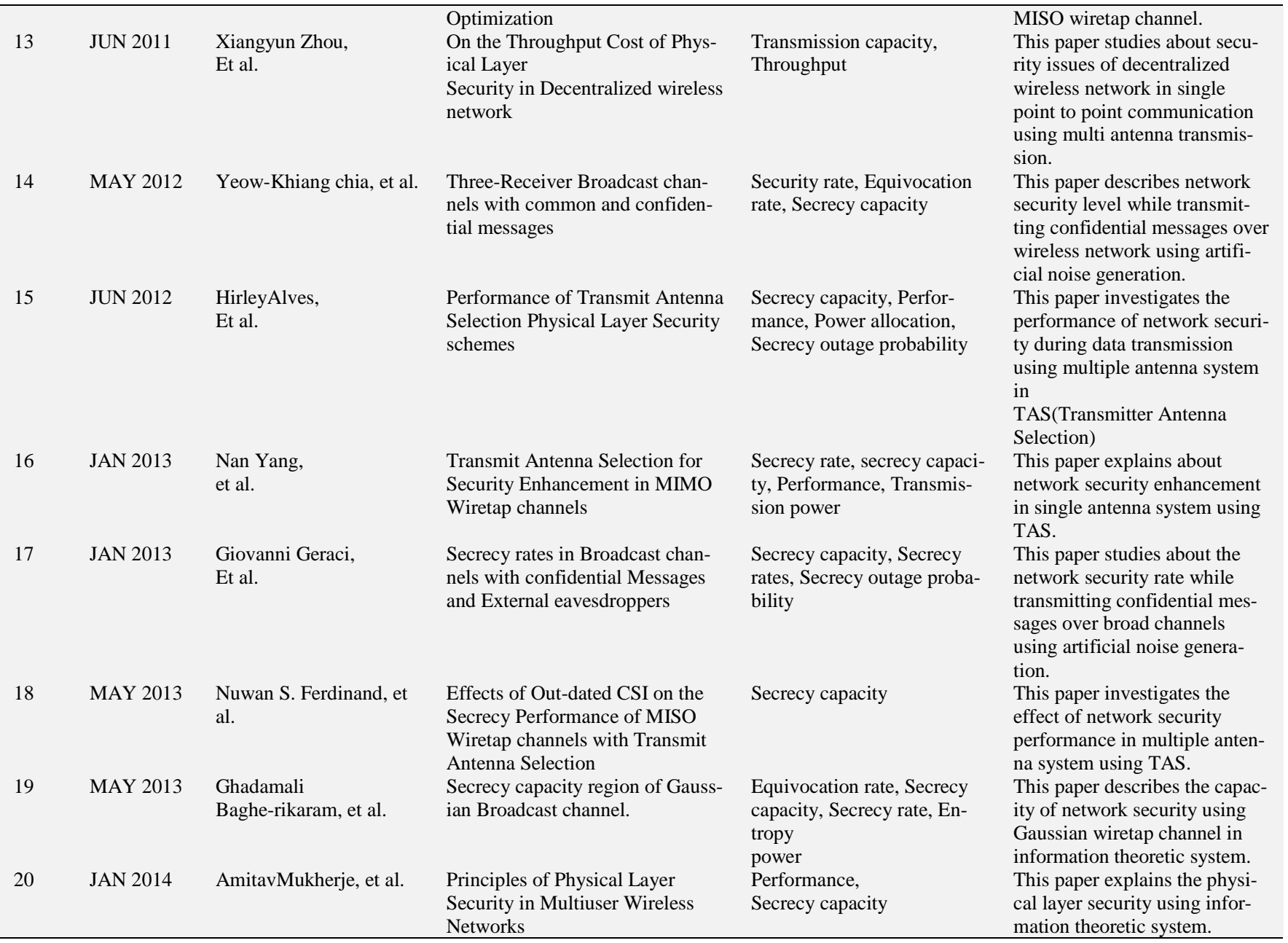

Table 2:. Measurements Taken in Aodv Routing Protcol

\begin{tabular}{|c|c|c|c|c|c|c|c|}
\hline$\overline{\mathrm{SNO}}$ & YEAR & AUTHOR & TOPIC & MEASUREMENTS & APPLICATIONS & $\begin{array}{l}\text { EXISTING SYS- } \\
\text { TEM }\end{array}$ & $\begin{array}{l}\text { PROPOSED } \\
\text { SYSTEM } \\
\end{array}$ \\
\hline \multirow[t]{2}{*}{1} & 2000 & $\begin{array}{l}\text { Elizabeth } \\
\text { M. Royer, } \\
\text { et al. }\end{array}$ & $\begin{array}{l}\text { An Implementation } \\
\text { study of the AODV } \\
\text { routing protocol }\end{array}$ & Routing overhead & Data routing & $\begin{array}{l}\text { Ad hoc proactive } \\
\text { routing protocol }\end{array}$ & $\begin{array}{l}\text { AODV } \\
\text { routing protocol }\end{array}$ \\
\hline & 2001 & $\begin{array}{l}\text { Sonali Bharga- } \\
\text { va, et al. }\end{array}$ & $\begin{array}{l}\text { Security Enhance- } \\
\text { ments in AODV } \\
\text { protocol for wireless } \\
\text { Ad hoc networks }\end{array}$ & $\begin{array}{l}\text { Routing load, } \\
\text { Packet delay, Accuracy, } \\
\text { Throughput, } \\
\text { Packet delivery } \\
\& \\
\text { Routing overhead }\end{array}$ & $\begin{array}{l}\text { Secured routing } \\
\text { in Military opera- } \\
\text { tions }\end{array}$ & $\begin{array}{l}\text { Multi hop routing } \\
\text { schemes }\end{array}$ & $\begin{array}{l}\text { AODV } \\
\text { routing protocol }\end{array}$ \\
\hline 3 & 2007 & $\begin{array}{l}\text { Asad Amir } \\
\text { Pirzada, } \\
\text { et al. }\end{array}$ & $\begin{array}{l}\text { Multi - linked } \\
\text { AODV Routing } \\
\text { protocol for wireless } \\
\text { Mesh network }\end{array}$ & $\begin{array}{l}\text { Performance, } \\
\text { Routing Overhead, } \\
\text { Interference rate } \\
\& \\
\text { Packet delivery }\end{array}$ & Routing & $\begin{array}{l}\text { Multi hop wireless } \\
\text { network }\end{array}$ & $\begin{array}{l}\text { AODV - ML } \\
\text { (Multi-Link) }\end{array}$ \\
\hline 4 & 2007 & $\begin{array}{l}\text { Asad Amir } \\
\text { Pirzada, } \\
\text { et al. }\end{array}$ & $\begin{array}{l}\text { High performance } \\
\text { AODV routing pro- } \\
\text { tocol for Hybrid } \\
\text { wireless Mesh Net- } \\
\text { work }\end{array}$ & $\begin{array}{l}\text { Packet delay, Through- } \\
\text { put } \\
\& \\
\text { Packet loss }\end{array}$ & Secured Routing & $\begin{array}{l}\text { Standard AODV } \\
\text { routing }\end{array}$ & $\begin{array}{l}\text { AODV - HP } \\
\text { (Hybrid Rout- } \\
\text { ing) }\end{array}$ \\
\hline 5 & 2008 & $\begin{array}{l}\text { Ahed M. Al- } \\
\text { shanyour, } \\
\text { Et al. }\end{array}$ & $\begin{array}{l}\text { Bypass AODV: } \\
\text { Improving Perfor- } \\
\text { mance of } \\
\text { Ad hoc On-demand } \\
\text { Distance Vector } \\
\text { (AODV) routing } \\
\text { protocol in wireless } \\
\text { Ad hoc Networks }\end{array}$ & $\begin{array}{l}\text { Node density, } \\
\text { Node mobility } \\
\& \\
\text { Performance }\end{array}$ & Routing & $\begin{array}{l}\text { AODV routing } \\
\text { (Unidirectional } \\
\text { routing) }\end{array}$ & $\begin{array}{l}\text { Bypass AODV } \\
\text { routing }\end{array}$ \\
\hline 6 & 2009 & $\begin{array}{l}\text { Mehdi Medadi- } \\
\text { ah, et al. }\end{array}$ & $\begin{array}{l}\text { Combat with Black } \\
\text { Hole attack in } \\
\text { AODV routing pro- } \\
\text { tocol in MANET }\end{array}$ & $\begin{array}{l}\text { Path rate, } \\
\text { Packet delivery } \\
\& \\
\text { Performance }\end{array}$ & Routing security & $\begin{array}{l}\text { Proactive routing } \\
\text { protocol } \\
\text {-DSDV } \\
\text { (Destination Se- } \\
\text { quence Distance } \\
\text { Vector) }\end{array}$ & $\begin{array}{l}\text { Reactive routing } \\
\text { protocol-AODV }\end{array}$ \\
\hline 7 & 2009 & $\begin{array}{l}\text { Hidehisa Naka- } \\
\text { yama, et al. }\end{array}$ & $\begin{array}{l}\text { A dynamic Anomaly } \\
\text { Detection scheme for } \\
\text { AODV - Based }\end{array}$ & $\begin{array}{l}\text { Projection distance } \\
\& \\
\text { Forgetting curve }\end{array}$ & $\begin{array}{l}\text { Secured routing } \\
\text { in Disaster, } \\
\text { Military }\end{array}$ & $\begin{array}{l}\text { ryptography with } \\
\text { proactive routing } \\
\text { protocol }\end{array}$ & $\begin{array}{l}\text { MANET routing } \\
\text { protocol } \\
\text {-Anomaly detec- }\end{array}$ \\
\hline
\end{tabular}




\begin{tabular}{|c|c|c|c|c|c|c|c|}
\hline & & & $\begin{array}{l}\text { mobile Ad hoc net- } \\
\text { works }\end{array}$ & & \& & & $\begin{array}{l}\text { tion: } \\
\text { AODV routing }\end{array}$ \\
\hline 8 & 2012 & $\begin{array}{l}\text { Mehdi Barati, } \\
\text { et al. }\end{array}$ & $\begin{array}{l}\text { Performance evalua- } \\
\text { tion of energy con- } \\
\text { sumption for AODV } \\
\text { and DSR routing } \\
\text { protocols in MANET }\end{array}$ & $\begin{array}{l}\text { Route delay, Routing } \\
\text { overhead, Packet deliv- } \\
\text { ery\&Performance }\end{array}$ & Secured routing & $\begin{array}{l}\text { Proactive routing } \\
\text { methods }\end{array}$ & $\begin{array}{l}\text { Reactive routing } \\
\text { (AODV } \\
\& \text { DSR } \\
\text { routing ) }\end{array}$ \\
\hline 9 & 2013 & Sheng Liu, et al. & $\begin{array}{l}\text { Research of AODV } \\
\text { routing protocol for } \\
\text { Ad hoc networks }\end{array}$ & $\begin{array}{l}\text { Performance, } \\
\text { Average delay, Routing } \\
\text { overhead } \\
\text { \& Packet delivery ratio }\end{array}$ & Routing repair & $\begin{array}{l}\text { Multi hop } \\
\text { Ad hoc networks }\end{array}$ & $\begin{array}{l}\text { AODV } \\
\text { routing protocol }\end{array}$ \\
\hline
\end{tabular}

Table 3: Algorithms Used in Existing Research Works recommendations for AODV Routing with Cryptographic Algorithm

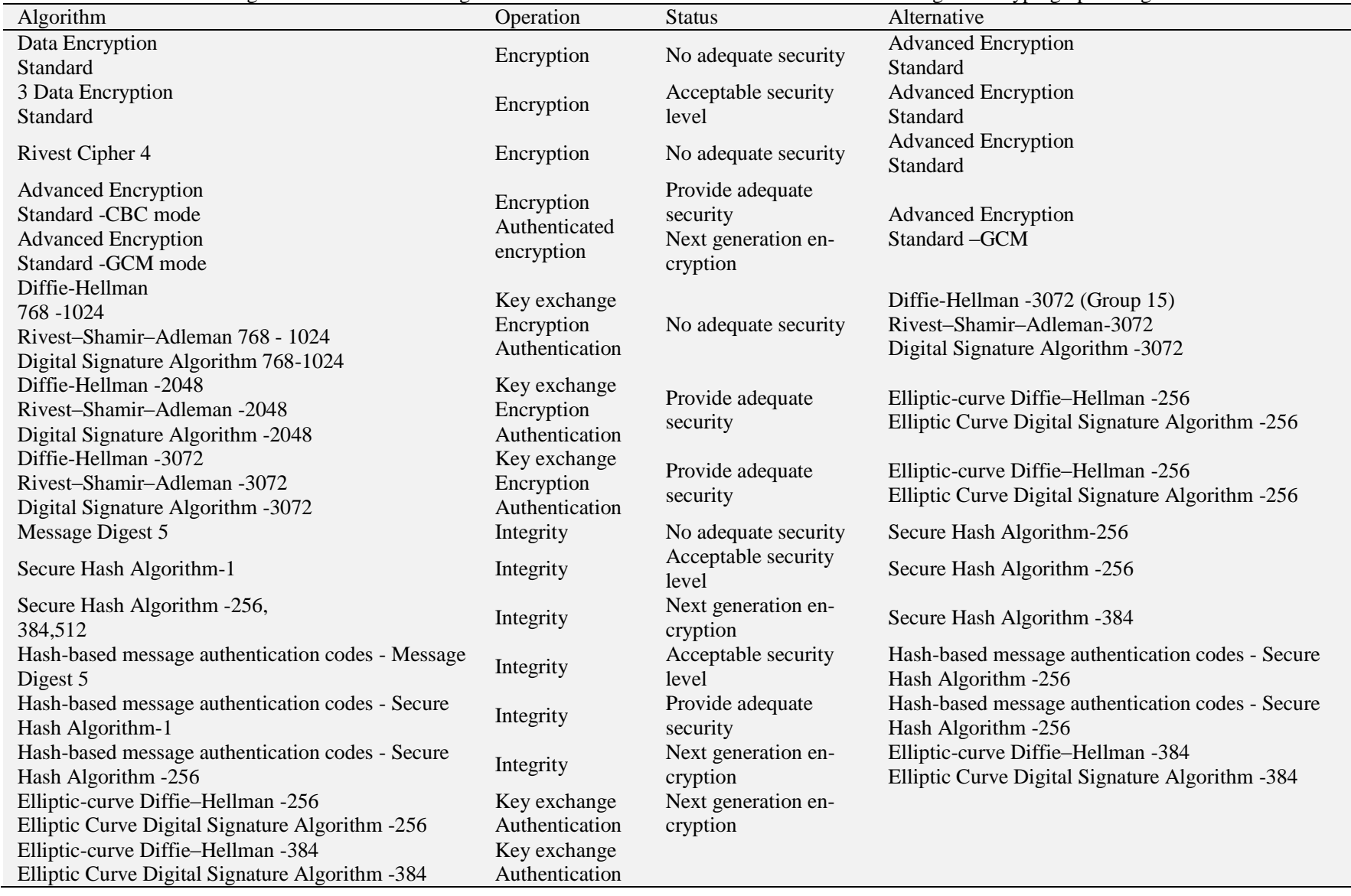

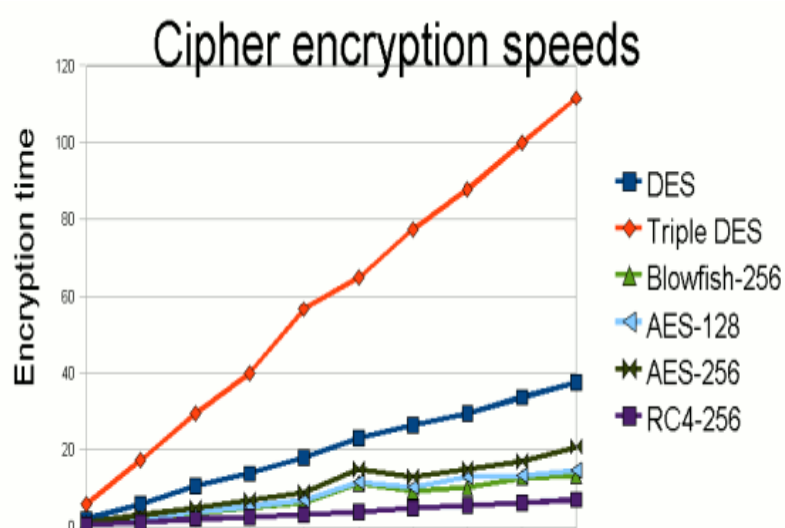

16004800800011200144001760020800240002720030400

No 16-byte blocks

Fig. 1: Speed Of Cryptographic Algorithm.

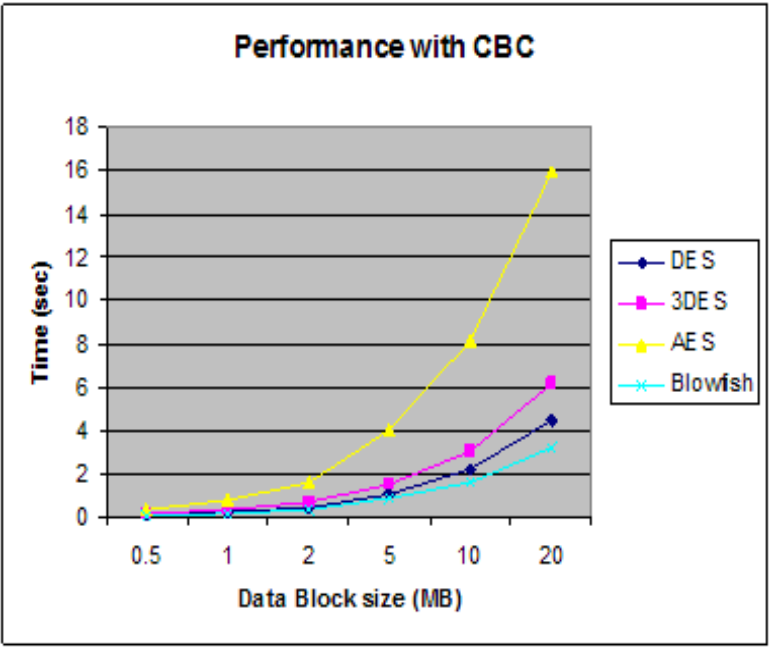

Fig. 2: Performance of Cryptographic Algorithm.

\section{Conclusion}

In this work, we suggested AODV-BA which deflects route breaks established on AODV in ad-hoc routing protocols. It is the princi- 
ple where each intervening node on an active route reveals the vulnerability of the interconnection break to the upstream node planted on four aspects of the received radio, the overlay of paths, the battery and consistency. The route breaks are avoided by restyling a prime route with our prospective innovation before the route breaks.

\section{References}

[1] A. D.Wyner, "Thewire-tapchannel,"Bell Syst.Tech. J., vol.54, no.8, pp. 1355-1387, 1975

[2] I. Csiszár and J. Körner, "Broadcast channels with confidential messages," IEEE Trans. Inf. Theory, vol. IT-24, no. 3, pp. 339-348, May 1978.

[3] R. Liu, I.Maric, P. Spasojevic, andR. D. Yates, "Discrete memory less interference and broadcast channels with confidential messages: Secrecy rate regions," IEEE Trans. Inf. Theory, vol. 54, no. 6 , pp. 2493-2507, Jun. 2008.

[4] G.Bagherikaram, A.S.Motahari, A.K.Khandani, "Securebroadcasting: The secrecy rate region," in Proc. 46th Annu. Allerton Conf. Commun., Control Comput. Sep. 2008, pp. 834-841.

[5] A. Khisti, A. Tchamkerten, and G. Wornell, "Secure broadcasting over fading channels," IEEE Trans. Inf. Theory, vol. 54, no. 6, pp. 2453-2469, Jun. 2008

[6] Royer, Elizabeth M., and Charles E. Perkins. "An implementation study of the AODV routing protocol." Wireless Communication and Networking Confernce, 2000.WCNC.2000 IEEE.Vol. 3. IEEE, 2000.

[7] Bhargava, Sonali, and Dharma P. Agrawal. "Security enhancements in AODV protocol for wireless ad hoc networks." Vehicular Technology Conference, 2001. VTC 2001 fall. IEEE VTS 54th.Vol. 4 IEEE, 2001.

[8] Gwalani, Sumit, Elizabeth M. Belding-Royer, and Charles E. Perkins. "AODV-PA: AODV with path accumulation." Communications, 2003.ICC'03.IEEE International Conference on.Vol. 1. IEEE, 2003.

[9] Pirzada, Asad Amir, Ryan Wishart, and Marius Portmann. "Multilinked AODV routing protocol for wireless mesh networks." Global Telecommunications 2007.GLOBECOM'07.IEEE.IEEE, 2007.

[10] Pirzada, Asad Amir, and Marius Portmann. "High performance AODV routing protocol for hybrid wireless mesh networks." Mobile and Ubiquitous Systems: Networking \& Services, 2007. MobiQuitous 2007.Fourth Annual International Conference on.IEEE, 2007.

[11] Medadian, Mehdi, Mohammad HosseinYektaie, and Amir MasoudRahmani. "Combat with Black Hole Attack in AODV routing protocol in MANET." Internet, 2009.AH-ICI 2009.First Asian Himalayas International Conference on.IEEE, 2009.

[12] Alshanyour, Ahed M., and UthmanBaroudi. "Bypass AODV: improving performance of ad hoc on-demand distance vector (AODV) routing protocol in wireless ad hoc networks." Proceedings of the first international conference on ambient media and systems. ICST (Institute for Computer Sciences, Social-Informatics and Telecommunications Engineering), 2008.

[13] Nakayama, H., Kurosawa, S., Jamalipour, A., Nemoto, Y., \& Kato, N. (2009). A dynamic anomaly detection scheme for AODV-based mobile ad hoc networks. IEEE transactions on vehicular technology, 58(5), 2471-2481.

[14] Barati, Mehdi, KayvanAtefi, FarshadKhosravi, and YasharAzabDaftari. "Performance evaluation of energy consumption for AODV and DSR routing protocols in MANET." In Computer \& Information Science (ICCIS), 2012 International Conference on, vol. 2, pp. 636-642.IEEE, 2012

[15] Liu, Sheng, Yang Yang, and Weixing Wang. "Research of AODV routing protocol for ad hoc networks1." AASRI Procedia 5 (2013): 21-31.

[16] ZhouLidong and Zygmunt J. Haas. "Securing ad hoc networks." IEEE network 13.6 (1999) 2430.

[17] Gupta, Piyush, and Panganmala R. Kumar. "The capacity of wireless networks." IEEE Transactions on information theory 46.2 (2000): 388-404

[18] Anand, Santhanakrishnan, and RajarathnamChandramouli. "On the location of an eavesdropper in multiterminal networks." IEEE Transactions on Information Forensics and Security 5, no. 1 (2010): 148-157

[19] Zhou, Xiangyun, and Matthew R. McKay. "Secure transmission with artificial noise over fading channels: Achievable rate and op- timal power allocation." IEEE Transactions on Vehicular Technology 59.8 (2010): 3831-3842.

[20] Pinto, Pedro C., João Barros, and Moe Z. Win. "Wireless secrecy in large-scale networks." Information Theory and Applications Workshop (ITA), 2011.IEEE, 2011.

[21] Huang, Jing, and A. Lee Swindlehurst. "Robust secure transmission in MISO channels based on worst-case optimization." IEEE Transactions on Signal Processing 60.4 (2012): 1696-1707.

[22] Chia, Yeow-Khiang, and Abbas El Gamal. "Three-receiver broadcast channels with common and confidential messages." IEEE Transactions on Information Theory 58.5 (2012): 2748-2765.

[23] Yang, N., Yeoh, P. L., Elkashlan, M., Schober, R., \&Collings, I. B. (2013). Transmit antenna selection for security enhancement in MIMO wiretap channels. IEEE Transactions on Communications, 61(1), 144-154.

[24] Ferdinand, Nuwan S., Daniel Benevides da Costa, and MattiLatvaaho. "Effects of outdated CSI on the secrecy performance of MISO wiretap channels with transmit antenna selection." IEEE Communications Letters 17.5 (2013): 864-867.

[25] Mukherjee, A., Fakoorian, S. A. A., Huang, J., \&Swindlehurst, A. L. (2014). Principles of physical layer security in multiuser wireless networks: A survey. IEEE Communications Surveys \& Tutorials, 16(3), 1550-1573.

[26] Deepa.A, Ramkumar.S," Active Resource and Job Allocation for Power Minimization in Mobile Cloud Systems", International Journal of Advanced Research Trends in Engineering and Technology, Vol.3 (12), pp.57-63, Mar-2016.

[27] Sibi.V, Ramkumar.S," Privacy Reserving in Multimedia Big Data Using Secure Data Repository System", International Journal of Advanced Research Trends in Engineering and Technology, Vol.3 (17), pp.01-10, Mar-2016. 